VII. Aus der ärztlichen Praxis.

\title{
1. Ein Fall von centralem Dammriss mit Entwickelung des
} Kindes durch denselben.

Von Dr. W. Succow in Gembitz.

Die anerkannte Seltenheit der vollkommenen centralen Dammrisse mit Entwickelung der Frucht durch dieselben, an deren Vorkommen man uberhaupt vor nicht allzulanger Zeit zweifelte und die namhafte Geburtshelfer selbst niemals beobachtet zu haben angeben, lässt eine Veröffentlichung jedes derartigen Falles ohne weiteres als berechtigt und wünschenswerth erscheinen, zumal wenn derselbe für die ätiologische Beurtheilung bemerkenswerthe Verhältnisse aufweist.

Auf Grund derartiger Erwägungen nehme ich Veranlassung, nachstehende eigene Beobachtung zur Kenntniss weiterer ärztlicher Kreise zu bringen. Ich konnte in dem betreffenden Fall nicht nur die Entstehung der seltenen Verletzung vollständig beobachten, sondern hatte auch nach Ablauf des Wochenbettes Gelegenheit, die erkrankte Frau in meiner Sprechstunde eingehender, als es bei der Entbindung thunlich war, auf den Bau des Beckens und die Bildung der Genitalien hin zu untersuchen. Wenn im Laufe der Darstellung hier und da der äusseren Umstände Erwähnung geschieht, so dürfte das Aussergewöhnliche derselben hinreichenden Grund dazu bieten.

Am 12. August 1894 früh $4 \mathrm{Uhr}$ wurde ich zur Hülfeleistung bei der Entbindung nach dem etwa $2^{1 / 2}$ Meilen von hier entfernt, dicht an der russischen Grenze gelegenen Dorfe 0 . gerufen, und zwar nach einem von dem eigentlichen Dorfe weit entfernten armseligen Gehöft. Die Kreissende war eine russische Polin von etwa 28 Jahren; sie lag in ihrer gewöhnlichen, bis zum freiwilligen Abfallen ohne Wechsel getragenen Kleidung auf dem Boden der elenden Hütte auf einer Streu, welche aus halb verfaultem Stroh und eben solchen darüber gebreiteten Lumpen bestand, und machte auf den ersten Anblick einen höchst erbärmlichen Eindruck. Eine Hebamme war angeblich nicht anfzutreiben gewesen. Zunächst liess ich ein Lager für die Frau in der Weise herrichten, dass auf einer starken, uber zwei feste Böcke gelegten Stallthür die nothwendigste weiche Unterlage durch Stroh und einige Kleidungsstücke hergestellt wurde, während ich fur die Unterlage des Beckens aus meinem fur solche Zwecke bereits mitgebrachten, ziemlich reichlichen Vorrath an Watte, desinficirtem Leinen und etwas wasserdichtem Stoffe sorgte. Die Einzelheiten der schwierigen und langwierigen Desinfection tibergehe ich, um die während derselben auf eingehendes Nachforschen von der Frau erlangten anamnestischen Daten zu geben. Die Kreissende war immer viel krank gewesen, hatte als Kind sehr schwer und spät gehen gelernt, später an einem "Typhus" und als junges Mädchen der Beschreibung nach an Chlorose mit andauernden Menstruationsstörungen gelitten. Die erste Entbindung machte sie mit 20 Jahren durch, später folgten noch zwei, die letzte vor drei Jahren. Sämmtlich waren sie sehr lange dauernd und schwer gewesen, besondes die erste, bei welcher ihr ein Arzt Beistand leistete. Während und nach der letzten Entbindung war sie durch sehr heftige Blutungen dem Tode nahe gewesen; die Kinder waren entweder todt zur Welt gekommen oder kurz nach der Geburt gestorben. Die jetzige Schwangerschaft glaubte sie, ohne bestimmte Angaben machen zu können, an ihrem normalen Ende angelangt. Wehen waren mit stundenlangen Unterbrechungen seit mehr als 24 Stunden eingetreten, auch Wasser war bereits in ziemlicher Menge abgegangen; seit dem frühen Morgen habe sie angeblich infolge Genusses eines selbstbereiteten Thees, uber dessen Bestandtheile ich nichts erfahren konnte, heftige, lange anhaltende und äusserst schmerzhafte Wehen bekommen, so dass sie jetzt vollkommen ermattet sei.

1) Zur Ausspülung von Fremdkörpern und Ceruninalpfröpfen ist eine grosse Spritze nøthig, die nicht in einem Etui untergebracht werden kann. Es ist dies auch nicht erforderlich, da man bei diesem Eingriff doch stets zu Hause operirt. 
Die äussere Untersuchung der kleinen, sehr welken und schwach bemuskelten Frau, welche doppelt so alt schien, als sie zu sein angah, ergab unzweifelhafte Zeichen fruhherer Rachitis; die Rippen waren verbogen, das Sternum stand hervor, die unteren Extremitäten waren auffallend kurz, die Gelenke aufgetrieben, die Unterschenkel verliefen in einem nach aussen convexen Bogen, und die oben und nach innen nicht zusammenschliessenden Oberschenkel liessen dicht unter Damm und Vulva eine weite Lücke klaffen. Dadurch trat ein anderer, sofort bei der äusseren Besichtigung ins Auge fallender Umstand noch auffallender hervor: es wurde die fast horizontal liegende und in Ruckenlage zum grossen Theil ohne weiteres vor Augen tretende Vulva vollends bis an den Damm sichtbar, und selbst von diesem sah man, zu Fiüssen der Kreissenden stehend, einen Theil. Auffallend war ferner die ungewöhnliche Länge des Dammes, dessen Messung leider erst später erfolgte. Ausserdem bestand Hängebauch mässigen Grades. Der Uterus war etwas nach rechts geneigt; der Rücken des Kindes links, kleinere Theile rechts fühlbar. Kindesbewegungen und Herztöne nicht wahrnehmbar; auch die Frau gab an, dass sie seit einiger Zeit keine Kindesbewegungen mehr empfunden habe. Den Kopf des Kindes fühlte ich deutlich beim Eingehen mit den Fingerspitzen dicht über der Symphyse. Der Contractionsring der Gebärmutter stand kaum drei Finger breit unter dem Nabel.

Von auffallender Beschaffenheit waren die äusseren Genitalien: Die grossen Schamlippen entbehrten durchaus der Turgescenz und standen als ein paar dünne, trockene, fast harte Hautlappen klaffend auseinander; zwischen ihnen ragten die vergrösserten, gleichfalls starren und etwas klaffenden kleinen Schamlippen weit hervor; der Scheideneingang war zwar nicht sehr eng, aber ausserordentlich rigide und wenig dehnbar die Scheide weit und faltenlos, ihre Schleimhaut der Feuchtigkeit und Schlüpfrigkeit entbehrend. Ich erinnere mich, solche Genitalien nur einige male bei offentlichen Dirnen nach sexuellen Erkrankungen, am allerwenigsten aber je bei Hochschwangeren oder Kreissenden gesehen zu haben. Uebrigens war der Muttermund verstrichen, die Blase geplatzt; der Kopf stand quer und fest im Beckeneingang, und zwar in Vorderscheitelbeineinstellung, die kleine Fontanelle weit nach links, die grosse rechts und tiefer stehend, die Pfeilnaht in der Nähe des. Promontoriums quer verlaufend. Der Kopf schien nicht gross zu sein. Bemerkenswerth war - worauf ich weiter unten noch zurückkomme - der fast ohne Concavität stark nach hinten gestreckte Verlauf der vorderen Kreuzbeinfläche, mit welcher das daran sich schliessende Steissbein einen scharf markirten Winkel bildete.

Bei dem Fehlen jeglichen Lebenszeichens seitens des Kindes und dem höchst elenden, wenn auch fieberfreien Zustande der Frau, besonders aber mit Rücksicht auf die drohende Gefahr der Gebärmutterzerreissung, welche das Aufrücken des Contractionsringes infolge der heftigen und vergeblichen Wehenthätigkeit (siehe unten) nahe legte, kam als Kunsthülfe hier kaum etwas anderes als die sofortige Perforation in Betracht: wenn auch die Aussicht, dieselbe ohne Narkose und ohne jede Assistenz als die einer alten schwachen Frau und des angetrunkenen Ehemannes ausfuihren zu müssen, keine ermuthigende war. So bereitete ich denn schnell alles auf dieselbe vor, versuchte jedoch im letzten Augenblick noch einmal, in einer der kurzen Wehenpausen durch Druck von oben den fest eingekeilten Kopf weiter herunter zu bringen. In der That gluckte diesmal der Versuch: Indem ich mit den Fingerspitzen beider Hände etwas seitlich von der Mittellinie dicht îber der Symphyse moglichst tief einging und besonders rechts auf das Vorderhaupt einen kräftigen Druck ausuibte, gelang es mir, unter deutlich vernehmbarem Ruck den Kopf in das kleine Becken hinunterzubringen.

Von diesem Augenblicke an verlief die Geburt schneller, als dass ich die Folgen des rapiden Verlaufes, selbst wenn dieselben vorauszusehen gewesen wären, hätte vereiteln können. Fast gleichzeitig mit dem Heruntertreten des Kopfes setzte eine ausserordentlich heftige, krampfartige Wehe ein, unter deren Qualen die Kreissende sich hin und her wand und, ohne auf mein eindringliches Verbot zu achten, mit aller Kraft ihre Bauchpresse in gewaltsame Thätigkeit setzte. Vergebens suchte ich rom Hinterdamm und, als das nicht gelang, unter Eingehen mit zwei Fingern der linken Hand vom After aus den Kopf, welcher den langen und dünnen Damm stark hervorwölbte, nach der Vulva hinzudrücken, während ich mit der rechten Hand von der Vagina aus den Kopf zurückzuhalten suchte; die Kreissende selbst vereitelte meine Bemuhungen durch das Hin- und Herwälzen sowie das krampfhafte Mitpressen. Als fast ohne Pause wiederum eine fast noch stürmischere Wehe eintrat, schmolz der halbkugelförmig vorgewölbte Damm plötzlich in der Mitte auseinander, und der Kopf des Kindes trat, das schmale Vorderhaupt voraus, durch die weit klaffende Wunde des Dammes zu Tage. Das Ganze verlief so schnell, dass mir nicht einmal Zeit blieb, meine im After der Gebärenden besudelten Finger zu reinigen. So entwickelte ich denn das Kind vollständig durch den Dammriss und nabelte es, da die Nabelschnur nicht pulsirte, sofort ab. Das Hinterhaupt des Kindes hatte sich jedenfalls seitlich angestemmt, während das schmale Vorderhaupt nach abwärts und vorn trat, sich dann aber auf dem langen Damm verfing and bei der stürmischen Wehenthätigkeit und dem starken Pressen der Frau tief in denselben wie in eine Hobhlung einbohrte, ohne nach der Vulva zu sich drehen zu können.

Die Lösung der Placenta trat, beschleunigt durch den Crédé'schen Handgriff, bald ein, und zwar erfolgte die vollständige Ausstossung derselben gleichfalls durch die gewaltige Scheidendammfistel.

Mit Rücksicht auf die bessere Versorgung und vor allem mit Rücksicht auf die, zu Hause entweder ganz ungenugende oder vollkommen mangelnde Nachbehandlung der Frau hätte ich dieselbe gern in ein Krankenhaus therwiesen. Indessen wusste ich aus mehrfacher Erfahrung, dass derartige Leute hier zu Lande thatsächlich auf keine Weise dazu zu bewegen sind, ein Krankenhaus aufzusuchen und lieber zı Hanse elend um- kommen, als diesen Schritt thun. Auch hier waren alle Vorstellungen vergebens, und so blieb nichts übrig, als ehne Narkose und ohne jede Assistenz an die sofortige Vernähung der stark blutenden Wunde zu gehen

Die Dammwunde begann hinter einem schmalen, höchstens $1 \frac{1 / 2}{2}$ bis $2 \mathrm{~cm}$ breiten Gewebsstreifen und zog sich bis in den After hinein, ohne jedoch den Sphincter ani vollständig zil durchsetzen. In der Scheide setzte sich der Riss in zwei Schenkeln fort, von denen der linke in einer Länge von ungefähr $6 \mathrm{~cm}$ neben der Columna rugarum posterior verlief und sich nach oben und aussen allmählich von derselben entfernte, während der rechte in nach oben convexem Bogen sich etwa bis zum Vereinigungs. punkt zwischen oberem und mittlerem Drittel der kleinen Schamlippe hinaufzog und $1 \mathrm{~cm}$ hinter diesem Punkte endete. Nach nochmaliger grüdlicher Reinigung und Desinfection durchtrennte ich, dem von Charpentier gegebenen und von F. Mathaei in der Deutschen medicinischen Wochenschrift 1894, No. 19 befïrworteten Rathe folgend, z1lnächst den nach der Scheide zu stehen gebliebenen, brückenartigen $\mathrm{Ge}$ websstreifen, so die ungeheure Scheidendammfistel in einen grossen, gewöhnlichen Dammriss verwandelnd. In der That ist so die genaue Orientirung auf dem Operationsfelde und die Uebersicht iuber die unregelmässigen, stellenweise arg zerfetzten Wundflächen ganz wesentlich erleichtert, ja dann erst eigentlich ermöglicht. Mehrfach musste ich durch Wegnehmen von zerquetschten Gewebsfetzen die ausgedehnten Wundflächen erst anpassungsfähig machen, ehe ich mit einer, unter den äusseren Umständen recht schwierigen, möglichst genauen Aneinanderlegung der Flächen und ganz besonders der Ränder die Naht vornehmen onnte.

Die Vernähung begann ich vom oberen Winkel der linken Vaginalwunde aus, um die ubrigen Wundflächen dabei vor Augen haben und in Bezug auf die Adaption auf sie gleich Rïcksicht nehmen zu können, nnd nähte mehrfach übereinander mit fortlaufendem Catgutfaden. Die zum Schluss erfolgende Vereinigung der Dammwunde liess das Gewebe derselben verschiedentlich als älteres Narbengewebe und stellenweise ansserordentlich brüchig erscheinen, so dass anch hier reichliche und weitgreifende Entspannungsnähte erforderlich waren.

Meine Befurchtung, die Frau werde durch starke Schmerzäusserungen die Naht sehr erschweren, erwies sich als ïbertrieben. Ihr waren, wie sie selbst mir gestand, von ihrem Manne während der letzten 24 Stunden so bedeutende Mengen scharfer und natïrlich sehr schlechter Alkoholica eingeflösst worden, dass nach dem endlichen Ueberstehen der Entbindung ihre Reactionsfähigkeit im wesentlichen erschöpft war und sie von den Schmerzen des Nähens nicht übermässig gequält wurde.

Die grösste Sorge erwuchs ans der doch so nothwendigen Nachbehandlung. Die hiesigen Verhältnisse - muss ich erläuternd bemerken - bringen es in solchen Fällen mit sich, dass der Arzt die Wöchnerin während des Wochenbettes meist nicht wiedersieht, wenn er nicht aus eigenem Antriebe und - bei der materiellen Lage dieser Lente - auch auf eigene Kosten den weiten Weg machen will. Dass dies beim besten Willen sich nicht regelmässig durchführen lässt, ist einleuchtend, nnd leider besuchen auch die Hebammen nach der Entbindung die Wöchnerinnen nicht wieder. In diesem Falle führte mich eigenes Interesse am nächsten Tage, der Zufall einige Tage später nach 0 ., resp. in dessen Nähe, und so konnte ich beobachten. dass der. Heilungsverlauf hier, wo doch yon dem Augenblicke meines Wegganges an gewiss mehr als reichliche Gelegenheit zur Infection gegeben war, ein vollkommen reactionsloser und glatter war.

Als nach einigen Wochen die Frau, meinem Wunsche entsprechend, zu mir in's Haus kam, konnte ich mich von der Vollständigkeit der Heilung üherführen und zugleich denjenigen Verhältnissen ihres Körperbaues meine Aufmerksamkeit noch einmal mit Musse zuwenden, welche für den abnormen Verlauf der Entbindung von Bedentung gewesen sein konnten.

Den Beckenmaassen der Frail setze ich die des kindlichen Schädels, welchen ich seinerzeit nach Anlegung der Nähte gleich einer bezüg-
lichen Betrachtung unterw orfen hatte, hier vorauf. Das Kind erschien zwar gut ausgebildet, war aber bedeutend kleiner, als es am normalen Ende der Entwickelung hätte sein miissen. Es war jedenfalls erst während der lange dauernden Entbindung abgestorben. Seine Länge betrug knapp $46 \mathrm{~cm}$; sein Gewicht konnte leider nicht festgestellt werden. Der Schädel war ziemlich stark in die Länge gezogen und seitlich comprimirt; auf dem etwas untergeschobenen rechten Stirnbein zeigte sich nahe der Kronennaht ein ausgeprägter, vom Promontorium herrührender Eindruck, das Vorderhaupt war besonders schmal. Die Maasse des Schädels waren folgende: Der Umfang betrug $32 \mathrm{~cm}$, der gerade Durchmesser $11 \frac{1}{2} \mathrm{~cm}$, der grosse quere Durchmesser $8 \frac{1}{2} \mathrm{~cm}$, der kleine quere Durchmesser 7 , der grosse schräge Durchmesser $121 / 2$, der kleine schräge Durchmesser $8 \mathrm{~cm}$. Somit waren sämmtliche Maasse des Schädels, theilweise nicht unbedeutend, kleiner als am normal entwickelten, reifen Kinde. Eine wesentliche Kopfgeschwulst hatte sich nicht gebildet.

Die Maasse des mutterlichen Beckens, mit dem Martin'schen Tasterzirkel gemessen, waren folgende: Diameter spinarum $=27 \mathrm{~cm}$; Diameter cristarum $=27 \mathrm{~cm}$; Conjugata externa $=18 \mathrm{~cm}$; Conjugata diagonalis $=12,5 \mathrm{~cm}$; Conjugata vera $=9,5 \mathrm{~cm}$. Die mehrmals vorgenommene Messung der Beckenneigung gegen den Horizont ergab ca. $39^{\circ}$, war also eine sehr geringe. Der Damm hatte nach der vollkommenen Verheilung eine Länge von $8 \mathrm{~cm}$.

Der Fall weist, mit Rücksicht auf die Aetiologie der Verletzung betrachtet, eine grosse Reihe von Momenten auf, welche für die Entstehung eines centralen Dammrisses begünstigend einwirken können und als begünstigend thatsächlich betrachtet werden. Alle diese Momente lassen sich nach ihrer grösseren oder geringeren Gewichtigkeit in zwei Gruppen theilen, in solche, welche das Zinstandekommen der Verletzing aberhaupt 
ermöglichen, für dasselbe eine unerlässliche Bedingung sind; und solche, welche nur die Rolle der begünstigenden Factoren spielen. $\mathrm{Zu}$ den ersteren möchte ich vor allem rechnen: eine bedeutende Länge des Damnies bei Schlaffheit und Schwäche seines Gewebes und einen rapiden, mit starker Wehenthatigkeit einhergehenden Verlauf der Austreibungsperiode. Was den ersten Punkt betrifft, so dürfte bei kurzem oder normalem Damm wohl immer nur Einreissen desselben vom Rande her stattfinden; reisst indessen der Damm hinter einer -stehenbleibenden Gewebebrücke ein, so entsteht, wenn die Möglichkeit des Durchtrittes der Frucht durch den Riss gegeben sein soll, eher eine ungeheure Mastdarmscheidenfistel als ein centraler Dammriss. Die bedeutende Länge des Dammes bedingt neist eine gewisse Dunne desselben, und wenn dazu noch Narbenl von früheren Entbindungen oder soustigen Verletzungen sich gesellen, so ist damit der Entstehung unserer Verletzung in hohem Grade Vorschub geleistet. Auf den rapiden Geburtsverlauf, die heftige Wehenthätigkeit und das Mitpressen der Kreissenden möchte ich ganz besonderen Werth legen, denn bei normalem, langsamem Verlauf der Entbindung liisst sich die Frucht wohl immer gentigend nach der Vulva zu dirigiren, unl ihr Festsitzen auf dem Damm zu verhindern. In unserem Falle wichen die Wehen vollkommen von der Norm ab: sie setzten von vornherein, ohme allmähliches Anschwellen, mit elementarer Gewalt ein, so zwar, dass das Mitpressen der Kreissenden weit mehr einer reflexartigen Schmerzäusserung als einer vom Wollen abhängigen Muskelaction ähnlich schien. Die Wehenpausen schwanden fast ganz, und die beiden Wehen, welche das Austreten der Frucht bewirkten, folgten einander ohne wesentliche Pause. Von allen ubrigen, mehr begünstigenden Momenten, Verlauf der hinteren Beckenwand, geringe Neigung des Beckens, Rigidität der Vulva, Grö́sse und Lage der Frucht etc. möchte ich am meisten Gewicht auf die Kleinheit der Frucht und deren Lage legen. Nur eine kleine Frucht gestattet im allgemeinen ein derartig schnelles Heruntertreten bis fest auf den Damm, dass man ein rechtzeitiges Abweichen nach der Vulva zu nicht mehr bewirken kann. Im allgemeinen werden - und mit Recht - die Beckenendlagen als begünstigend für das Zustandekommen des centralen Dammrisses betrachtet; kommt derselbe aber bei Schädellagen zustande, so dirfte keine andere derselben als Herabtreten des schmalen Vorderhauptes auf den Damm gtinstiger sein, ganz besonders aber bei kleinem Kopf. Dieser Kindeslage und Configuration des Kopfes, welcher durch den lange andauernden Druck des Promontoriums auf das rechte Stirnbein und Unterschieben des letzteren unter das linke noch besonders nach vorn zu verschmälert war, fällt auch in unserem Falle die Rolle eines stark begïnstigenden Momentes zu. Allen anderen Verhältnissen lege ich, für den vorliegenden Fall wenigstens, einen weniger hohen Werth bei; leider stand mir, um die Aetiologie der centralen Dammrisse im allgemeinen hier zu berücksichtigen, nicht die nöthige Litteratur zu Gebot, so dass ich mich in der Beziehung lediglich auf die eine eigene Beobachtung beschränken muss. 